\title{
PRIMER REGISTRO DE AGAVE SISALANA (AGAVACEAE, ASPARAGALES) PARA COLOMBIA
}

\author{
Diego Giraldo-Cañas
}

Herbario Nacional Colombiano (COL), Instituto de Ciencias Naturales, Universidad Nacional de Colombia, Bogotá D. C., Colombia; dagiraldoc@unal.edu.co (autor corresponsal).

\begin{abstract}
Giraldo-Cañas, D. 2020. First report of Agave sisalana (Agavaceae, Asparagales) for Colombia Darwiniana, nueva serie 8(2): 490-498.

Agave sisalana Perrine, a native species from Yucatán Peninsula (Mexico), is recorded for the first time for Colombia. It is morphologically related to Agave fourcroydes Lem. and Agave pax GiraldoCañas, but clearly differs from it in various vegetative and reproductive characteristics, which are given. Thus, a total of ten species of Agave are currently known in South America, seven are native and three are naturalized. A key for the South American species is included.
\end{abstract}

Keywords. Agave; Agavaceae; Asparagales; Flora of Colombia; sisal.

Resumen. Giraldo-Cañas, D. 2020. Primer registro de Agave sisalana (Agavaceae, Asparagales) para Colombia. Darwiniana, nueva serie 8(2): 490-498.

Se registra, por primera vez para Colombia, a Agave sisalana Perrine, una especie nativa de la península de Yucatán (México). Esta especie está morfológicamente relacionada con Agave fourcroydes Lem. y Agave pax Giraldo-Cañas, pero difiere claramente de éstas por varias características vegetativas y reproductivas, las cuales se presentan. Así, el género Agave queda representado en la flora sudamericana por diez especies, siete nativas y tres naturalizadas. Se presenta una clave para reconocer las especies presentes en Sudamérica.

Palabras claves. Agave; Agavaceae; Asparagales; Flora de Colombia; sisal.

\section{INTRODUCCIÓN}

Agave L. es un género americano, distribuido desde el sur de los Estados Unidos de América hasta Argentina (García-Mendoza, 2007, 2011; Gentry, 1982; Giraldo-Cañas, 2017). En Bolivia y Argentina, sólo se hallan dos especies cultivadas y naturalizadas (A. americana L. y A. salmiana Otto ex Salm-Dyck); en Brasil solo se halla una especie ampliamente cultivada (A. sisalana Perrine) (Giraldo-Cañas, 2020) y naturalizada en varios estados (e.g. Bahia, Paraíba, Pernambuco).
De este género se conocen aproximadamente 265 especies (Thiede, 2020a), siendo México su centro de mayor riqueza y endemismo (GarcíaMendoza et al., 2019), particularmente en el valle de Tehuacán-Cuicatlán en la región limítrofe entre los estados de Oaxaca y Puebla (Thiede, 2020a). En Sudamérica sólo se habían documentado nueve especies, de las cuales seis son endémicas.

Muchas de las especies de Agave se usan de muy variadas maneras desde tiempos precolombinos hasta la actualidad (véanse los usos y las citas en Giraldo-Cañas, 2017). Entre estos usos, se tiene la 
producción de fibras de gran calidad, y una de las especies más destacadas para tal fin, es el sisal, $A$. sisalana, la cual es un cultígeno de México (TrejoTorres et al., 2018). De hecho, las fibras extraídas de $A$. sisalana suponen un $70 \%$ de la producción mundial de fibras duras (Guillot Ortiz et al., 2009). A esta especie se le encuentra cultivada en varios continentes (Martínez, 1988; Guillot Ortiz et al., 2009; Trejo-Torres et al., 2018), y en ocasiones, se le puede hallar escapada de cultivo e incluso, puede naturalizarse (Badano \& Pugnaire, 2004; Guillot Ortiz et al., 2009). Aquí se registra, por primera vez, a $A$. sisalana para Colombia, en el marco del estudio taxonómico-morfológico y el inventario de las especies de Agave, tanto nativas como naturalizadas y cultivadas, presentes en Sudamérica. Además, con este nuevo registro, se contribuye con el conocimiento de la flora de Colombia, considerada la segunda más diversa del mundo, después de la de Brasil (Rangel-Ch., 2006).

\section{MATERIALES Y MÉTODOS}

Se siguió la escritura del autor del binomio y la consideración del material tipo de A. sisalana, de acuerdo con lo expuesto por Trejo-Torres et al. (2018). Por lo tanto, siempre se va a referir como A. sisalana Perrine, y no como A. sisalana Perrine ex Engelm. Por su parte, se siguió a Nyffeler \& Eggli (2020) y Thiede \& Eggli (2020), para el reconocimiento y la circunscripción de la familia Agavaceae, así como su ubicación en el orden Asparagales. La circunscripción y la delimitación del género Agave están basadas en Thiede (2020a). Para referirse a los eventos de proliferación vegetativa de las inflorescencias (falsa viviparía), se emplea el término bulbilo y no bulbillo (véase Arizaga \& Ezcurra, 1995). Las medidas de las diferentes estructuras vegetativas corresponden exclusivamente a plantas en floración. Se siguió el concepto morfológico de especie (véase Giraldo-Cañas, 2017). Todas las determinaciones taxonómicas de los agaves, así como las de los materiales recolectados para la caracterización florística del hábitat de $A$. sisalana, fueron realizadas por el autor y los originales de las colecciones se encuentran depositados en COL, HUA, FMB y VALLE (Thiers, 2020).

\section{RESULTADOS Y DISCUSIÓN}

Agave sisalana Perrine, Trop. Pl. 87-88 (8-9, 16, 47, 60, 86). 1838. TIPO: México: Campeche, sin fecha, H. Perrine s.n. (Lectotipo: NY!, designado por J. C. Trejo-Torres, G. D. Gann \& M. J. M. Christenhusz. 2018. The Yucatán Peninsula is the place of origin of sisal (Agave sisalana, Asparagaceae): historical accounts, phytogeography and current populations. Botanical Sciences 96 (2): 366-379). Figs. 1 y 2.

Rosetas robustas, gregarias, rizomatosas, rosetas individuales hapaxánticas, 1,5-3,3 $\mathrm{m}$ de diámetro, con hijuelos rizomatosos, cortamente caulirrósula; tallo simple, $40-50(-145) \mathrm{cm}$ de alto $\times 18-25(-35) \mathrm{cm}$ de diámetro; raíces fibrosas, delgadas, numerosas; follaje denso (desde 50 hasta 101 hojas por roseta). Hojas espiraladas, ensiformes a linear-lanceoladas, lisas; márgenes inermes (muy raramente con diminutos y escasos dientes en hojas basales, caso en el cual éstos se ubican en la porción proximal de las hojas), sésiles, gruesas, coriáceas, rígidas, fibrosas, glabras, ligeramente concávas, dispuestas en ángulos de $50-60^{\circ}$, verde oscuras y opacas en ambas caras, 100-200 × 7-12 cm; venación inconspicua; espina terminal rígida, lisa, estriado-acanalada en la base, las estrías poco profundas, negruzca, rojiza o castaño oscura, opaca; hojas senescentes proximales persistentes. Inflorescencia terminal, una panícula laxa elíptica, bracteada y bracteolada, florífera, bulbilífera sólo cuando ha perdido las flores (bulbilos numerosos, vigorosos, verde-oscuros y opacos), (4,5-) 5-7 m de alto (incluido el pedúnculo); pedúnculo $10-14 \mathrm{~cm}$ de diámetro en su porción proximal, verde claro, opaco, de corteza dura que desprende en largas tiras; pedúnculo y ramificaciones primarias con tejido interno central esponjoso, blanquecino y opaco; porción fértil del pedúnculo a partir de la mitad de éste, así que desde la mitad hacia abajo es sólo bracteado, raramente el pedúnculo se hace fértil a partir del último tercio; pedúnculo con (16-) 22-29 ramificaciones primarias fértiles, estriadas longitudinalmente, espiraladas y distanciadas entre sí; ramificaciones cimosas constituidas por unidades monocasiales, las cuales dan la apariencia de umbelas; ramificaciones primarias proximales $45-60 \mathrm{~cm}$ long., las proximales con 8-9 ramificaciones 
secundarias cada una; ramificaciones verde-opacas; 1-3 (-4) flores por cada ramificación de último orden; brácteas proximales de hasta $120 \mathrm{~cm}$ long., largamente lanceoladas y acuminadas, raramente deltoides, divergentes o plegadas con respecto al pedúnculo, sin ramificaciones floríferas, las demás deltoideas, mucho más cortas y plegadas al pedúnculo, éstas van disminuyendo en tamaño a medida que se asciende en la inflorescencia; brácteas inermes, verde-claras y opacas, con un listón marginal crema a cada lado; bractéolas rugosas, café oscuras, opacas, generalmente deltoides, raras veces cortamente ensiformes o ligeramente falcadas, reduciéndose en longitud a medida que avanza el orden de ramificación, siempre más cortas que las flores; pedicelos verde claros y opacos, 0,3-0,9 (-1) cm long.; desarrollo acrópeto de las flores en el pedúnculo.
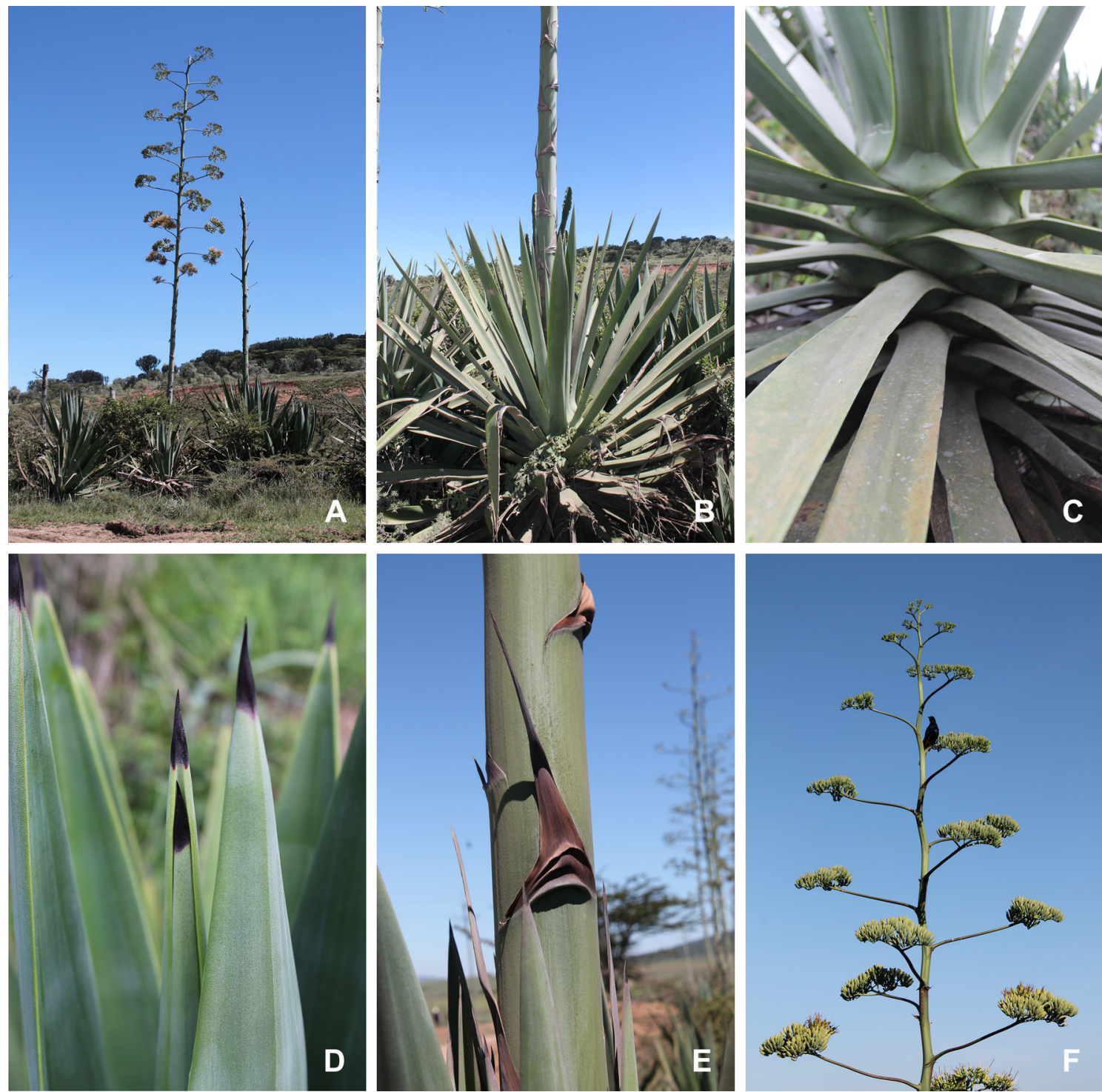

Fig. 1. Agave sisalana. A, hábito y hábitat. B, roseta florífera. C, porción proximal de las hojas. D, porción distal de las hojas y espinas foliares. E, bráctea proximal. F, arquitectura de la inflorescencia en preantesis. Fotografías: D. GiraldoCañas. Figura en color en la versión en línea http://www.ojs.darwin.edu.ar/index.php/darwiniana/article/view/910/1195 


\section{GIRALDO-CAÑAS. Primer registro de Agave sisalana para Colombia}
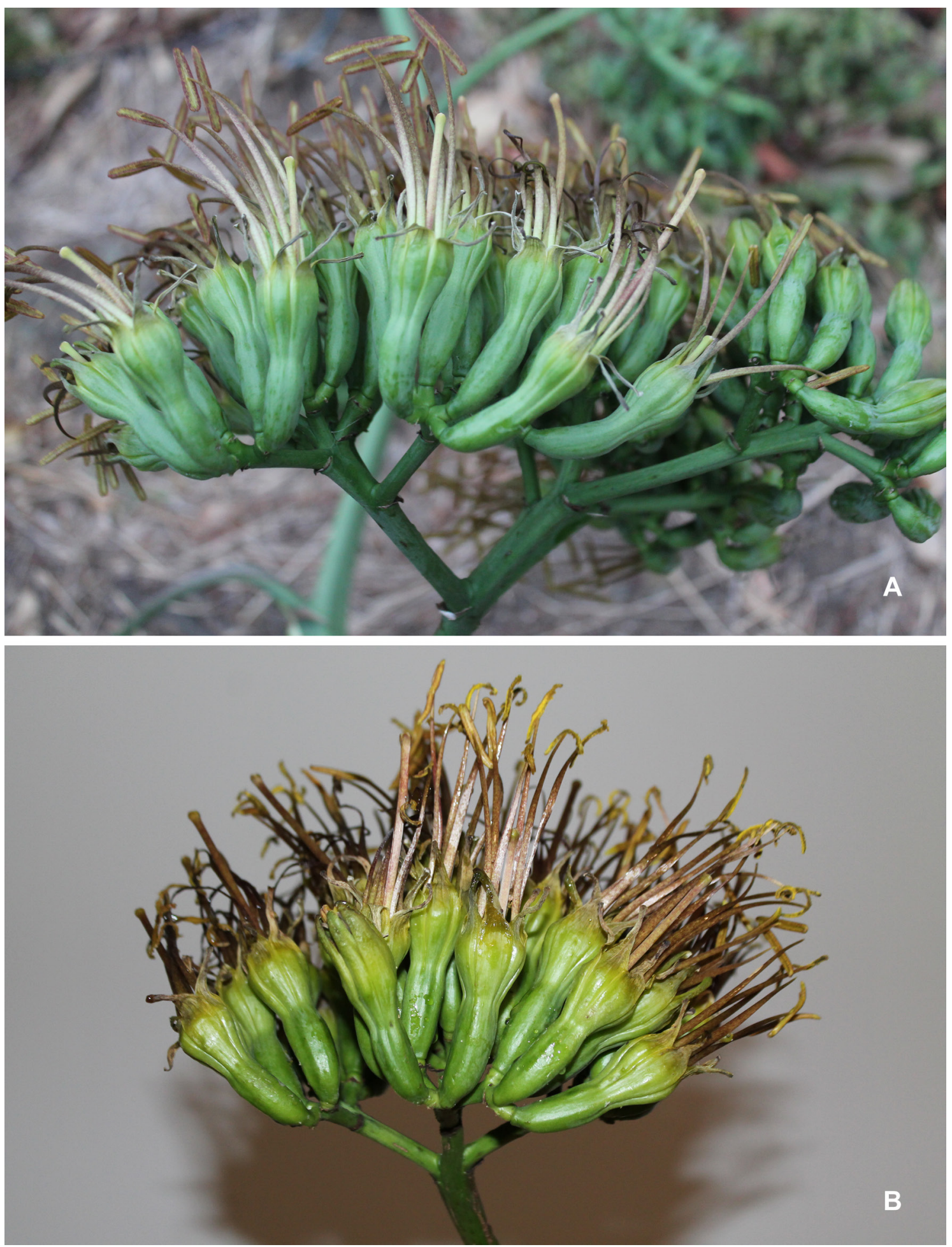

Fig. 2. Agave sisalana. A, flores de una ramificación media. B, flores de una ramificación distal. Fotografías: D. GiraldoCañas. Figura en color en la versión en línea http://www.ojs.darwin.edu.ar/index.php/darwiniana/article/view/910/1195 
Flores actinomorfas, hipocrateriformes, perfectas, gamotépalas, epíginas, protándricas, numerosas, densas, erectas, lisas, sinfragancias, pediceladas; ovario ínfero, tricarpelar, trilocular, 1,5-2,5 × 0,8-0,9 cm, suculento, verde-oscuro, ligeramente lustroso; placentación axilar; óvulos numerosos, anátropos, en dos series por lóculo; cuello inexistente a diminuto; tubo del perianto 1,5-1,8 cm long., suculento, verde-oscuro, ligeramente lustroso; tépalos 6 , basalmente fusionados (constituyendo el tubo del perianto), similares, linear-lanceolados, longitudinalmente estriados, 1,7-1,8 cm long., glabros, en su porción media-distal verde-cobrizos, mientras que en su porción proximal amarilloverdosos; lóbulos de los tépalos rectos a ligeramente arqueados, ensanchados en la base, largamente acuminados en su porción distal, delgados a escariosos, generalmente café claros, translúcidos, sin ornamentos, glabros; estilo filiforme, 7-8 cm long., largamente exerto, cobrizo-nacarado; estigma 3-lobado, papiloso, ca. 0,28-0,30 cm de diámetro, amarillo-crema; estambres 6 , adnatos \pm a la mitad del tubo; filamentos lineares, (6,5-) 7,5-8 cm long., amarillos, rojos o rojizos a cobrizonacarados, maculados, las máculas oscuras; anteras 2,2-2,7 cm long., lineares, dorsifijas, versátiles, café a amarillas, amarillo-cobrizas o verde-claras con matices granates o maculadas; polen amarillo, abundante; néctar muy abundante; flores visitadas por numerosas abejas y aves. Frutos no vistos (ningún individuo de la región presentaba frutos).

Etimología. El epíteto específico sisalana, proviene de la palabra sisal, nombre dado a las fibras extraídas de esta especie y a su vez, este nombre viene de la lengua maya zizal xui, nombre de una población de la península de Yucatán, de donde es originaria esta especie (Guillot Ortiz et al., 2009).

Nombres populares. En Colombia (Valle del Cauca) recibe los nombres de agave, fique, penca, mientras que en los demás países se le denomina agave y más frecuentemente, sisal.

Usos. En varios países se usa como planta ornamental y como cercas vivas o delimitación de linderos de propiedades, mientras que en otros, se emplea para la producción, a gran escala, de fibras duras (sisal, principalmente en Brasil y Kenia), las cuales se usan para hacer sogas, sacos (costales), empaques y diversas artesanías, generalmente, alfombras y cestería. En Brasil, los desechos de la producción de su fibra, resultantes del desfibrado de las hojas, se emplean para alimentar a los ganados bovino y caprino, como materia orgánica para enriquecer los suelos de los cultivos en áreas secas y en la elaboración de placas para la construcción de edificaciones.

Distribución y hábitat. Agave sisalana es originaria de la península de Yucatán (México), y de ahí se llevó a muchos países con fines de cultivo para la producción de fibra (Guillot Ortiz et al., 2009; Trejo-Torres et al., 2018), especialmente a Brasil (comienzos del siglo $\mathrm{xx}$ ) y los ingleses la llevaron a sus colonias africanas entre los años 1836 y 1842, en donde establecieron grandes cultivos (Martínez, 1988). Se trata de un taxón con claro carácter invasor del medio natural, una condición que ya había sido destacada por Guillot Ortiz et al. (2009) y confirmada aquí para el cañón de los ríos Dagua y Bitaco (departamento del Valle del Cauca, Colombia; Fig. 3). En esta localidad, la especie es muy frecuente y constituye una población abundante en ambas vertientes en este cañón, en una longitud de $17 \mathrm{~km}$, llegando a desplazar a las rosetas de Agave wallisii Jacobi [ $D$. Giraldo-Cañas \& S. D. Espinel-Galván 6178, 6180 (COL, FMB, HUA, VALLE)], un microendemismo colombiano, exclusivo de este cañón de bosques secos y cardonales (Cactaceae columnares).

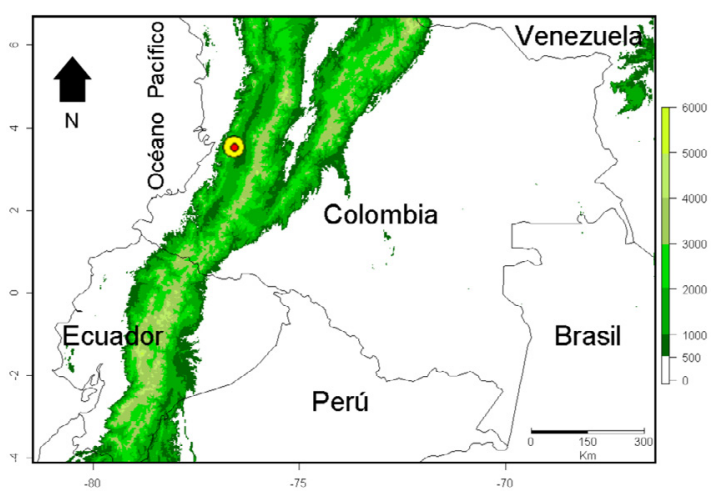

Fig. 3. Distribución de Agave sisalana en Colombia. La escala de colores representa la altura sobre el nivel del mar. Figura en color en la versión en línea http://www.ojs. darwin.edu.ar/index.php/darwiniana/article/view/910/1195 
Agave sisalana no había sido citada para Colombia, ni como especie cultivada ni como un elemento naturalizado(Bernal \& Gradstein, 2016). A pesar de ser una especie ampliamente cultivada para la producción de fibras en muchas regiones tropicales de ambos hemisferios y frecuentemente naturalizada (Guillot Ortiz et al., 2009), es curioso que en Colombia nunca se haya cultivado para tal fin y quizás sea porque en este país, desde tiempos precolombinos hasta la fecha, siempre se ha preferido cultivar diversas especies de fique (Furcraea Vent.) para la producción de fibras a gran escala (Pérez Mejía, 1964; Ullrich, 1992; Cadefique, 2006), principalmente $F$. cabuya Trel., $F$. foetida (L.) Haw. y F. selloana K. Koch (obs. pers.). De hecho, según Thiede (2020b: 333), la fibra obtenida de Furcraea, constituye la fibra nacional en Colombia. Esto nos ayudaría a entender la no incorporación de A. sisalana en la producción de fibras en Colombia, toda vez que en este país siempre han abundado y siguen abundando las poblaciones de varias especies de Furcraea a lo largo y ancho del territorio nacional. Además, desde tiempos precolombinos se había establecido y desarrollado todo el sistema de cultivo, cosecha, obtención de la fibra y sus diversos usos, por parte de diferentes etnias en Colombia. Así que esta especie pudo haber sido introducida al país, durante el auge a nivel mundial de su uso para la producción de fibras (finales del siglo xIX y comienzos del siglo xx) (véanse Martínez, 1988; Guillot Ortiz et al., 2009; Trejo-Torres et al., 2018), aunque dada la preferencia de los productores colombianos por las fibras de fique (Furcraea spp.), el cultivo de A. sisalana no prosperó en Colombia, por lo tanto, quedarían algunas plantas cultivadas en la región del cañón de los ríos Dagua y Bitaco, las cuales, después de un poco más de un siglo, colonizarían dicho cañón.

Agave sisalana prefiere las formaciones vegetales abiertas de áreas secas y los sustratos arenosos, pedregosos e incluso, rocosos, entre el nivel del mar y los $2800 \mathrm{~m}$ de altitud. En Colombia, se encontró una gran y dinámica población de esta especie en un cañón estrecho de los ríos Dagua y Bitaco (750-1000 m de altitud), en el departamento del Valle del Cauca, un área de escasas precipitaciones constituída por bosques secos, cardonales, arbustales xerofíticos y pastizales, sujetos a una fuerte alteración antrópica (extracción de madera, leña y materiales para la construcción, ganadería, cultivos a pequeña escala, obras civiles, parcelaciones y ampliación de las construcciones urbanas, entre otras). Esta área presenta suelos superficiales y pedregoso-arenosos, fácilmente erosionables, con pendientes de $45-60^{\circ}$, en la cual el componente arbóreo está caracterizado, principalmente, por Astronium graveolens Jacq., Bursera graveolens (Kunth) Triana \& Planch., Clusia cf. columnaris Engl., Enterolobium cyclocarpum (Jacq.) Griseb., Ficus sp., Guarea guidonia (L.) Sleumer, Guazuma ulmifolia Lam., Gyrocarpus americanus Jacq., Maclura tinctoria (L.) Steud., Muntingia calabura L., Ochroma pyramidale (Lam.) Urb., Pseudosamanea guachapele (Kunth) Harms y Zanthoxylum cf. formiciferum (Cuatrec.) P.G. Waterman; mientras que los arbolitos y los arbustos más frecuentes son Clibadium cf. grandifolium S.F. Blake, Cnidoscolus tubulosus (Müll. Arg.) I.M. Johnst., Cordia sp., Croton ferrugineus Kunth, Heliotropium fruticosum L., Jatropha gossypiifolia L., Solanum sp., Vachellia farnesiana (L.) Wight \& Arn., Vernonanthura brasiliana (L.) H. Rob. y Xanthium spinosum L.; por su parte, el componente herbáceo está caracterizado por Agave sisalana, A. wallisii, Anthurium cf. glaucospadix Croat, Aristida ternipes Cav., Arundinella hispida (Humb. \& Bonpl. ex Willd.) Kuntze, Bouteloua repens (Kunth) Scribn., Digitaria californica (Benth.) Henrard, Digitaria insularis (L.) Fedde, Epidendrum cf. fimbriatum Kunth, Furcraea selloana, Pitcairnia sp. y Tillandsia spp.; en todo el cañón había una destacada presencia de varias Cactaceae (Hylocereus megalanthus (Vaupel) Ralf Bauer, Melocactus curvispinus Pfeiff., Opuntia spp. y Stenocereus humilis (Britton \& Rose) D.R. Hunt).

Comentarios. Agave sisalana es fenotípicamente similar a las especies $A$. fourcroydes Lem. y A. pax Giraldo-Cañas, una especie endémica de Antioquia, Colombia, pero éstas se pueden diferenciar por varias características, tanto vegetativas como reproductivas, las cuales se detallan en la Tabla 1 . Agave fourcroydes es una especie cuyo ancestro silvestre es A. angustifolia Haworth (Colunga-García Marín et al., 1999; Thiede, 2020a), la cual también es ampliamente cultivada en diferentes países para la producción de fibras, conocida como henequén (Guillot Ortiz et al., 2009; Thiede, 2020a), incluso en los cultivos de ésta se suele mezclar con $A$. sisalana (Guillot Ortiz et al., 2009). Los sinónimos para $A$. sisalana se pueden consultar en Thiede (2020a). 
Con este nuevo registro, el género Agave queda representado en la flora sudamericana por diez especies, siete nativas y tres naturalizadas, nueve de las cuales están en Colombia. Además, se encuentran algunas especies cultivadas como $A$. americana, A. angustifolia, A. cf. applanata Hort. Tonel ex K. Koch, A. attenuata Salm-Dyck, $A$. colorata Gentry, A. desmetiana Jacobi, A. filifera Salm-Dyck, A. fourcroydes, A. macroacantha Zuccarini, A. parrasana A. Berger, A. cf. parryi Engelmann, A. salmiana, A. sisalana, A. tequilana F.A.C. Weber, A. victoriae-reginae T. Moore, y Agave sp. (Giraldo-Cañas, datos inéditos).

\section{Material examinado}

COLOMBIA. Valle del Cauca. Vertiente occidental de la cordillera Occidental andina, límites entre los municipios de Dagua y Restrepo, cañón de los ríos Dagua y Bitaco, carretera Buenaventura-Buga, a 15 kilómetros del peaje de Loboguerrero, cerca de Mediacanoa, suelos superficiales y pedregoso-arenosos, fácilmente erosionables, pendientes de $45-60^{\circ}$, bosques secos, cardonales, arbustales xerofíticos y pastizales, $c a$. $910 \mathrm{~m}$ de altitud, 348'55,6”N-76³6'13,3'O, 29-III-2018, D. Giraldo-Cañas \& S. D. EspinelGalván 6177, 6179 (COL, FMB, HUA, VALLE).

\section{Clave para diferenciar las especies nativas y naturalizadas de Agave L. presentes en Sudamérica}

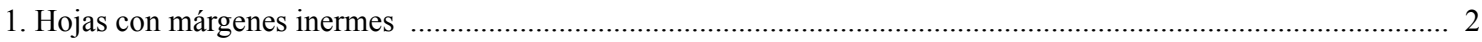

1. Hojas con márgenes conspicuamente armadas ........................................................................................ 3

2 (1). Hojas papilosas en ambas caras, lanceoladas-subuladas; espina terminal ligeramente rugosa, con numerosos puntos principalmente en su porción proximal; ovario 2,3-3,2 ×0,4-0,8 cm; cuello 0,30-0,40 × 0,25-0,45 cm; tubo (0,5-) 0,8-1,6 cm long.; tépalos largamente acuminados, 2,5-3 cm long., rala e irregularmente pilosos en su extremo distal; estilo 5-5,5 cm long.; estambres adnatos a la porción proximal de los lóbulos de los tépalos, a 0,30-0,32 cm del tubo; filamentos filiformes, 4,5-5,5 cm long.; anteras 1,4-1,6 cm long.

A. pax Giraldo-Cañas

2. Hojas carentes de papilas, ensiformes a linear-lanceoladas; espina terminal lisa, estriado-acanalada en la base, las estrías poco profundas; ovario 1,5-2,5 $\times 0,8-0,9 \mathrm{~cm}$; cuello inexistente a diminuto; tubo 1,5-1,8 cm long.; tépalos linear-lanceolados, 1,7-1,8 cm long., glabros; estilo 7-8 cm long.; estambres adnatos \pm a la mitad del tubo; filamentos lineares, (6,5-) 7,5-8 cm long.; anteras 2,2-2,7 cm long.

A. sisalana Perrine

3 (2). Panículas densas, comprimidas; hojas oblongo-ovadas, planas (muy raramente ligeramente cóncavas); ejes de las ramificaciones de la inflorescencia aplanados A. sylvesteriana Giraldo-Cañas

3. Panículas abiertas, laxas; hojas lanceoladas, linear-lanceoladas, oblongo-elípticas, espatuladas o estrechamente oblanceoladas (nunca oblongo-ovadas), cóncavas; ejes de las ramificaciones de la inflorescencia teretes ................ 4 4 (3). Rosetas no surculosas ................................................................................ A. cundinamarcensis A. Berger

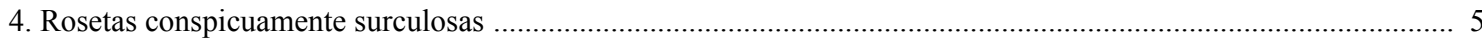

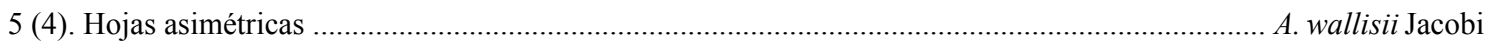

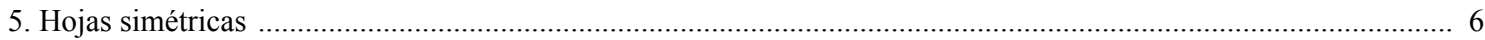

6 (5). Hojas con conspicuos listones longitudinales amarillentos en ambas caras ............................... A. americana L.

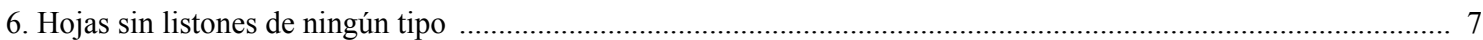

7 (6). Inflorescencias no bulbilíferas; flores 7,3-11 cm long.; frutos 5,5-8 cm long. ........................................... 8

7. Inflorescencias bulbilíferas; flores $4,3-5,8 \mathrm{~cm}$ long.; frutos $3,3-5 \mathrm{~cm}$ long. .................................................... 9

8 (7). Tépalos con lóbulos iguales en longitud; ovario 2,3-2,8 cm long., ligeramente arqueado; anteras 2-2,8 cm long.; hojas con espina terminal 2-3,5 cm long.; inflorescencia de hasta $16 \mathrm{~m}$ de alto .............. A. cordillerensis Lodé \& Pino 8. Tépalos con lóbulos desiguales en longitud, los externos un poco más largos que los internos; ovario 5-6 cm long., cilíndrico; anteras 3-3,5 cm long.; hojas con espina terminal 5-10 cm long.; inflorescencia de hasta $8 \mathrm{~m}$ de alto ..........

A. salmiana Otto ex Salm-Dyck

9 (7). Inflorescencia 4-5 m de alto; hojas estrechamente oblanceoladas; tépalos con lóbulos 1,5-2 $\times 0,4 \mathrm{~cm}$; frutos 3,3-3,6 cm long. ................................................................................................................ A. boldinghiana Trel.

9. Inflorescencia 6-9 $\mathrm{m}$ de alto; hojas lanceolado-espatuladas; tépalos con lóbulos 2-2,5 $\times 0,5-0,7 \mathrm{~cm}$; frutos 3,8-5 cm long. A. cocui Trel. 
D. GIRALDO-CAÑAS. Primer registro de Agave sisalana para Colombia

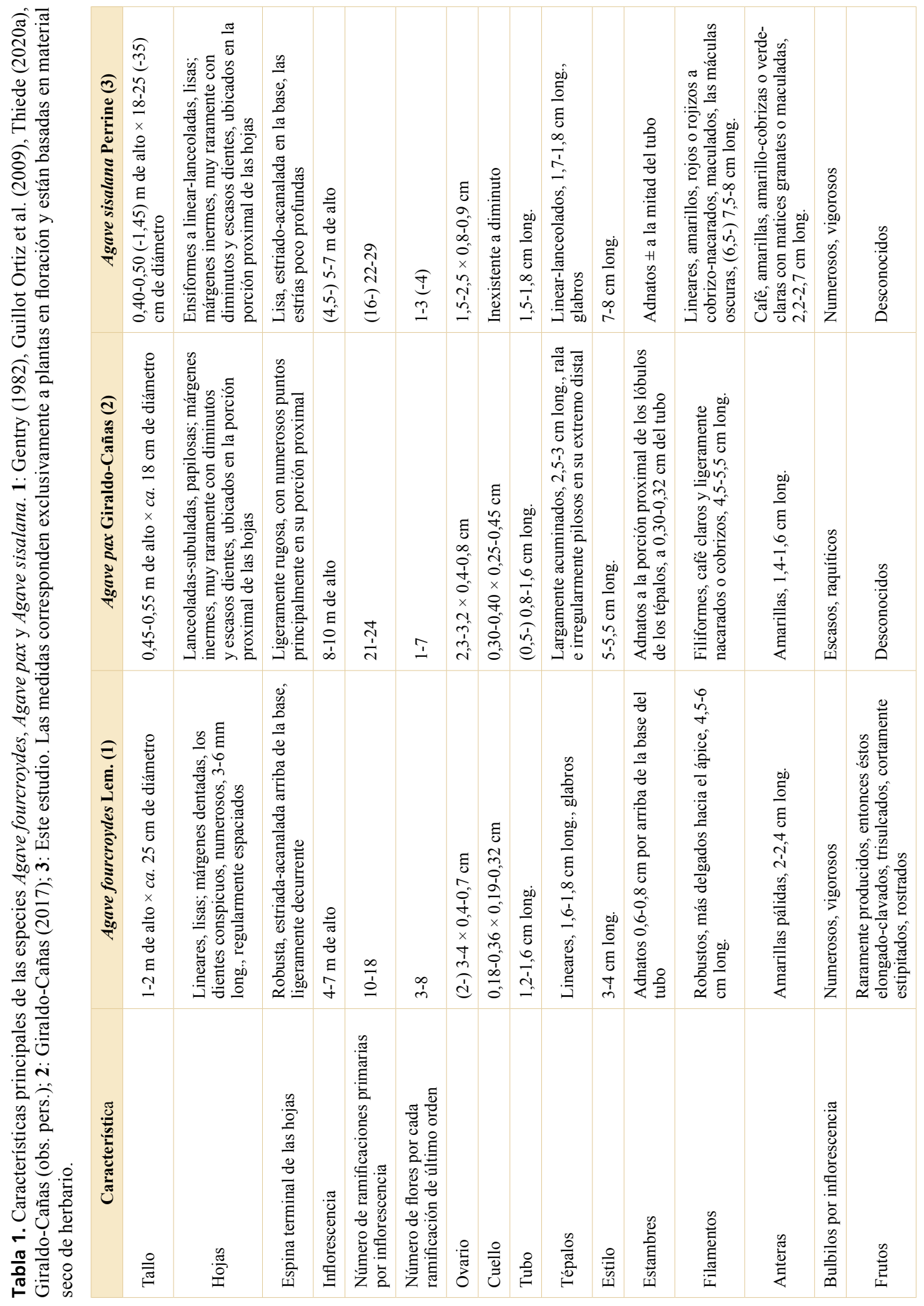




\section{AGRADECIMIENTOS}

Esta contribución es derivada de los proyectos "Estudios taxonómicos y morfológicos en los géneros Agave y Furcraea (Asparagaceae) en Sudamérica" (código Hermes 37417) y "Estudios florísticos en comunidades xerófilas de la Cordillera Oriental andina de Colombia" (código Hermes 26215), de la Universidad Nacional de Colombia, sede Bogotá. Al Dr. Abisaí GarcíaMendoza (MEXU), por sus valiosos comentarios, grata colaboración y por el obsequio de preciada bibliografía. A los biólogos Cristian Pinzón (Xalapa, México) y Daniel Díaz Rueda (COL), por el obsequio de numerosos materiales bibliográficos y fotografías. A Samuel David Espinel Galván y Jusset Sabagh por su grata colaboración en el trabajo de campo. Al Dr. Guillermo Pino Infante (Jardín Botánico de Lima, Perú), por compartir valiosa bibliografía y por el regalo de varios individuos vivos de diferentes especies de Agave y Furcraea. Al Prof. Edwin Trujillo (Universidad de la Amazonia, Florencia, Colombia) y al ingeniero Óscar Perdomo (Universidade Federal do Rio Grande do Sul, Porto Alegre, Brasil), por la elaboración del mapa. Al comité editorial, así como a los evaluadores, por sus acertados comentarios.

\section{BIBLIOGRAFÍA}

Arizaga, S. \& E. Ezcurra. 1995. Insurance against reproductive failure in a semelparous plant: Bulbil formation in Agave macroacantha flowering stalks. Oecologia 101: 329-334. DOI: https://doi.org/10.1007/BF00328819

Badano, E. I. \& F. I. Pugnaire. 2004. Invasion of Agave species (Agavaceae) in south-east Spain: invader demographic parameters and impacts on native species. Diversity and Distributions 10: 493-500.

Bernal, R. \& S. R. Gradstein. 2016. Asparagaceae, en R. Bernal, S. R. Gradstein \& M. Celis (eds.), Catálogo de plantas y líquenes de Colombia I, pp. 794-795. Bogotá D. C., Universidad Nacional de Colombia.

Cadefique (Cadena Productiva Nacional del Fique). 2006. Guía ambiental del subsector fiquero. Bogotá D. C.: Ministerio de Ambiente-Ministerio de Agricultura.

Colunga-García Marín, P.; J. Coello-Coello, L. E. Eguiarte \& D. Piñero. 1999. Isozymatic variation and phylogenetic relationships between henequén (Agave fourcroydes) and its wild ancestor A. angustifolia (Agavaceae). American Journal of Botany 86: 115-123.

García-Mendoza, A. J. 2007. Los agaves de México. Ciencias 87: $14-23$.

García-Mendoza, A. J. 2011. Agavaceae. Flora del Valle de Tehuacán-Cuicatlán 88: 1-95.
García-Mendoza, A. J.; I. S. Franco Martínez \& D. Sandoval Gutiérrez. 2019. Cuatro especies nuevas de Agave (Asparagaceae, Agavoideae) del sur de México. Acta Botánica Mexicana 126: e1461. DOI: https://doi. org/10.21829/abm126.2019.1461

Gentry, S. H. 1982. Agaves of Continental North America. Tucson: The University of Arizona Press.

Giraldo-Cañas, D. 2017. Una nueva especie de Agave (Asparagaceae) de Colombia y una clave taxonómica para las especies sudamericanas. Caldasia 39: 33-49. DOI: https://doi.org/10.15446/caldasia.v39n1.63318

Giraldo-Cañas, D. 2020. Una especie nueva de Agave (Asparagaceae: Agavoideae) de Colombia. Revista Mexicana de Biodiversidad 91: e913274. DOI: https://doi. org/10.22201/ib20078706e.2020.91.3274

Guillot Ortiz, D.; P. Van der Meer, E. Laguna Lumbreras \& J. A. Rosselló Picornell. 2009. El género Agave L. en la flora alóctona valenciana. Monografias de la revista Bouteloua 3: 1-91.

Martínez, M. Á. 1988. Contribuciones iberoamericanas al mundo. Botánica, medicina, agricultura. Madrid: Ediciones Anaya S. A.

Nyffeler, R. \& U. Eggli. 2020. Introduction to the classification of Monocotyledons, en U. Eggli \& R. Nyffeler (eds.), Illustrated handbook of succulent plants. Monocotyledons, pp. 1-6. Berlín: Springer-Verlag GmbH Germany, part of Springer Nature. DOI: https://doi.org/10.1007/978-3-662-56486-8

Pérez Mejía, J. A. 1964. El fique. Su taxonomía, cultivo y tecnología. Medellín: Compañía de Empaques-Editorial Colina.

Rangel-Ch., J. O. 2006. La biodiversidad de Colombia. Palimpsesto 5: 292-304.

Thiede, J. 2020a. Agave-Agavaceae, en U. Eggli \& R. Nyffeler (eds.), Illustrated handbook of succulent plants. Monocotyledons, pp. 21-311. Berlín: Springer-Verlag GmbH Germany, part of Springer Nature. DOI: https://doi. org/10.1007/978-3-662-56486-8

Thiede, J. 2020b. Furcraea-Agavaceae, en U. Eggli \& R. Nyffeler (eds.), Illustrated handbook of succulent plants. Monocotyledons, pp. 323-347. Berlín: Springer-Verlag GmbH Germany, part of Springer Nature. DOI: https://doi. org/10.1007/978-3-662-56486-8_106

Thiede, J. \& U. Eggli. 2020. Agavaceae, en U. Eggli \& R. Nyffeler (eds.), Illustrated handbook of succulent plants. Monocotyledons, pp. 9-19. Berlín: Springer-Verlag GmbH Germany, part of Springer Nature. DOI: https://doi. org/10.1007/978-3-662-56486-8

Thiers, B. (2020 [continuously updated]) Index Herbariorum: A global directory of public herbaria and associated staff. New York Botanical Garden's Virtual Herbarium. Available from: https://sweetgum.nybg.org/ih/

Trejo-Torres, J. C.; G. D. Gann \& M. J. M. Christenhusz. 2018. The Yucatán Peninsula is the place of origin of sisal (Agave sisalana, Asparagaceae): historical accounts, phytogeography and current populations. Botanical Sciences 96: 366-379. DOI: https://doi.org/10.17129/botsci.1928

Ullrich, B. 1992. Furcraea (Agavaceae) en Sudamérica. Quepo 6: 67-75. 